This item was submitted to Loughborough's Research Repository by the author.

Items in Figshare are protected by copyright, with all rights reserved, unless otherwise indicated.

\title{
Convergence behaviours of an adaptive step-size constant modulus algorithm for DS-CDMA receivers
}

PLEASE CITE THE PUBLISHED VERSION

PUBLISHER

(c) IEEE

VERSION

VoR (Version of Record)

LICENCE

CC BY-NC-ND 4.0

\section{REPOSITORY RECORD}

Yuvapoositanon, Peerapol, and Jonathon Chambers. 2019. "Convergence Behaviours of an Adaptive Stepsize Constant Modulus Algorithm for DS-CDMA Receivers". figshare. https://hdl.handle.net/2134/5914. 
This item was submitted to Loughborough's Institutional Repository (https://dspace.lboro.ac.uk/) by the author and is made available under the following Creative Commons Licence conditions.

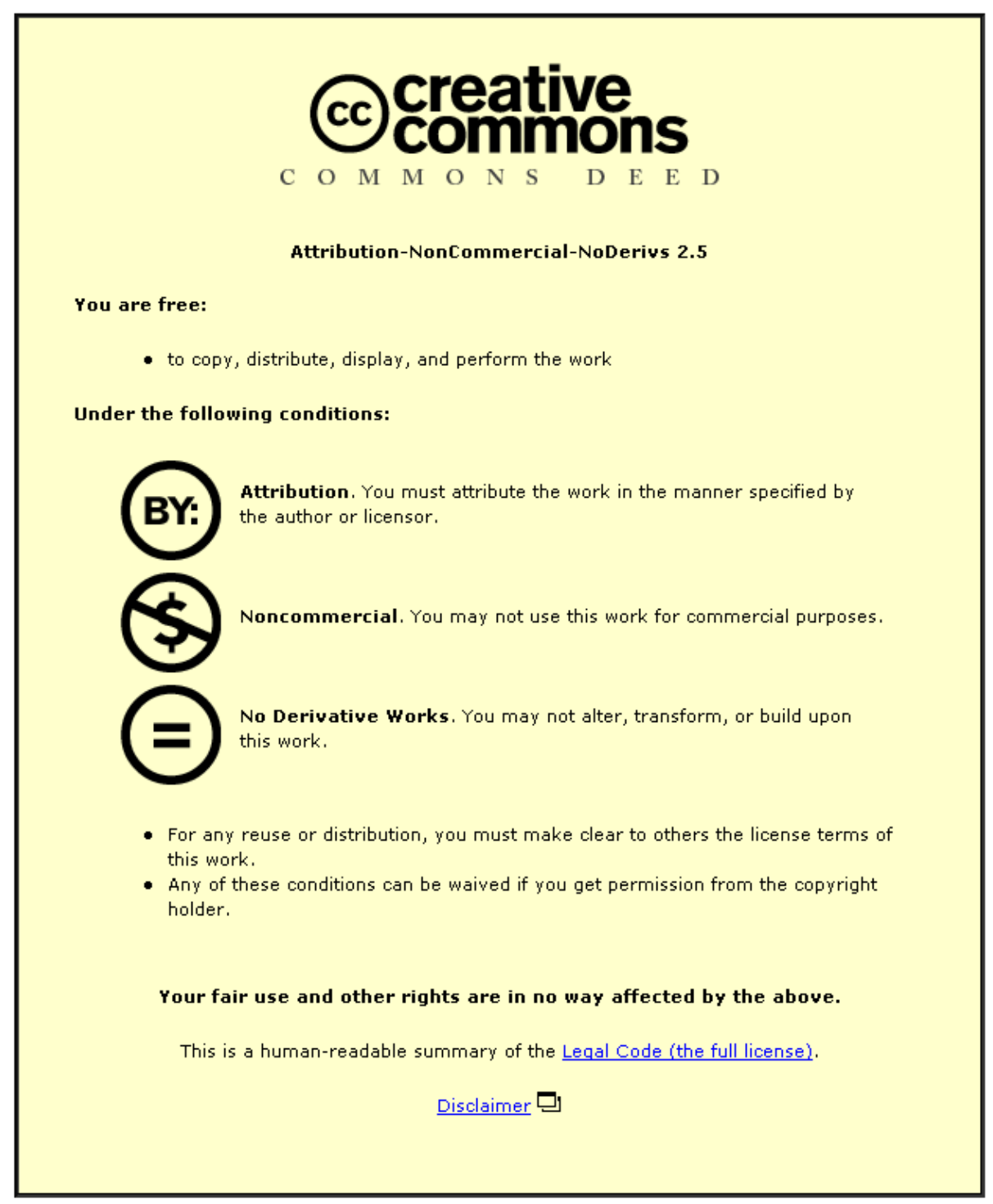

For the full text of this licence, please go to: http://creativecommons.org/licenses/by-nc-nd/2.5/ 


\section{CONVERGENCE BEHAVIOURS OF AN ADAPTIVE STEP-SIZE CONSTANT MODULUS ALGORITHM FOR DS-CDMA RECEIVERS}

\section{Peerapol Yuvapoositanon}

\author{
Department of Electronic Engineering, \\ Mahanakorn University of Technology, \\ Nong-Chok, Bangkok 10530, Thailand \\ Email: peerapol@mut.ac.th
}

\section{Jonathon A. Chambers}

\section{ABSTRACT}

A convergence analysis of the blind adaptive step-size constant modulus algorithm (AS-CMA) for direct-sequence code division multiple access (DS-CDMA) receivers is presented. Analytical results show similar convergence behaviours of the AS-CMA receiver and the adaptive step-size stochastic approximation (ASSA) receiver. Simulations show that the blind AS-CMA algorithm performs comparably with the non-blind AS-SA in a Markovian type of nonstationary environment. The robustness of the proposed algorithm to different settings of the initial step-size is also shown.

\section{INTRODUCTION}

An adaptive step-size constant-modulus algorithm (AS-CMA) has been proposed for a DS-CDMA receiver operating in nonstationary environments $[1,2]$. The algorithm adaptively varies the stepsize in order to minimise the constant modulus (CM) criterion. In contrast to the adaptive step-size minimum output energy (ASMOE) receiver proposed in [3], AS-CMA can be applied successfully in multipath fading CDMA channels [1]. This is because the MOE criterion is not designed to equalise the channel and tends to cancel the desired signal itself when the spreading waveform has a mismatch [4]. In reality, the multipath distortion, the cause of mismatch, must not be neglected especially when the transmission of high data rate is required.

In this paper, we study the convergence behaviours of the ASCMA receiver in multipath fading nonstationary channels as compared to the adaptive step-size stochastic approximation (AS-SA) receiver [5]. Further analysis from [1] reveals similarity in convergence behaviours of the $Y_{n}$ process, the derivative of the tapweight of with respect to the step-size, of AS-CMA and AS-SA.

Simulation results show that the performance in terms of signalto-interference plus noise ratio (SINR) of AS-CMA is comparable to that of the non-blind AS-SA receiver. Insensitivity of initial step-size settings is also confirmed. The trajectories of the stepsizes of AS-CMA are shown to behave similarly to those of AS-SA while AS-CMA enjoys the advantage of not requiring a training sequence for the adaptation process.

\section{SIGNAL MODEL}

Consider the real signal model of an additive white Gaussian noise (AWGN) $K$-user synchronous DS-CDMA channel, the baseband received signal is defined as

$$
r(t)=\sum_{i=-\infty}^{\infty} \sum_{k=1}^{K} A_{k} b_{k}(i) c_{k}(t-i T)+w(t),
$$

where $A_{k}$ represents the received amplitude of the $k$ th user. The data bits $b_{k}(i)$ are independent identically distributed (i.i.d.) and $b_{k}(i) \in\{-1,+1\}$. The symbol period is denoted by $T$. The spreading waveform of the $k$ th user $c_{k}(t)$ is $N$-dimensional and has unit energy property, i.e., $\left\|c_{k}\right\|_{2}^{2}=1$. The AWGN $w(t)$ has power spectral density $\sigma_{w}^{2}$. The spreading $\operatorname{codes} c_{k}(t)$ can be modified to take into account the effect of the channel and the pulse shaping waveform as $\bar{c}_{k}(t)=c_{k}(t) * \psi(t) * h_{k}(t)$, where $*$ denotes convolution, $\psi(t)$ is the pulse shaping filter and $h_{k}(t)$ is the chan. nel response of the $k$ th user and need not be identical for different users. The continuous-time received signal $r(t)$ is sampled to form a length- $L_{f}$ received signal vector at the $n$th observation, where $L_{f}$ is the length of a receiver with tap-weight vector $\mathbf{f}$,

$$
\mathbf{r}_{n}=\sum_{k=1}^{K} \mathbf{r}_{n}^{(k)}+\mathbf{w}_{n}=\sum_{k=1}^{K} \mathbf{G}_{k} \mathbf{b}_{k}[n]+\mathbf{w}_{n},
$$

where $\mathbf{G}_{k}$ is the combined code-channel response matrix of the $k$ th user and $\mathbf{b}_{k}[n]=\left[b_{k}\left[n+L_{b}-1\right], \cdots, b_{k}[n]\right]^{T}$ with $L_{b}=$ $\left\lceil\frac{L_{f}+L_{h}-1}{N}\right\rceil$ and $\mathbf{w}_{n}=\left[w\left[n N+L_{f}-1\right], \cdots, w[n N]\right]^{T}$. Note that

$\mathbf{G}_{k}=\mathbf{C}_{k} \mathbf{H}_{k}$
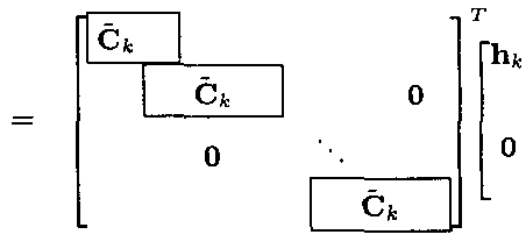

$\left.\begin{array}{r}\mathbf{0} \\ \mathbf{h}_{k}\end{array}\right]$,

where $\quad \tilde{\mathbf{C}}_{k}$ represents the block of delayed copies of the code sequence of the $k$ th user with dimension $L_{h} \times N+L_{h}-1$

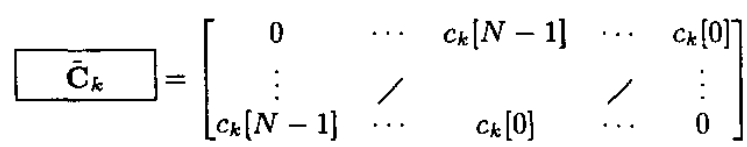

and $\overline{\overline{\mathbf{C}}_{k}}$ denotes the code matrix $\overline{\mathbf{C}}_{k}$ $L_{h}-1$ columns truncated. The channel response vector for the 
$k$ th user has length $L_{h}$, i.e., $h_{k}=\left[h_{0}^{(k)}, \cdots, h_{L_{h}-1}^{(k)}\right]^{T}$. For a linear receiver $f$, the estimate of the transmitted data $b[n]$ can be achieved as

$$
\hat{b}[n]=z_{n}=\mathbf{f}^{T} \mathbf{r}_{n}=\mathbf{f}^{T}\left(\mathbf{G b}[n]+\mathbf{w}_{n}\right),
$$

where $\mathbf{G}=\left[\mathbf{G}_{1}, \mathbf{G}_{2}, \cdots, \mathbf{G}_{K}\right]$ and $\mathbf{b}[n]=\left[\mathbf{b}_{1}^{T}[n], \mathbf{b}_{2}^{T}[n], \cdots\right.$, $\left.\mathbf{b}_{K}^{T}[n]\right]^{T}$. For brevity, we shall consider the first user as the desired user and drop the subscript $k$ in all variables involving the first user.

\section{ADAPTIVE STEP-SIZE CMA ALGORITHM}

We re-introduce the AS-CMA algorithm as presented in [1, 2] Consider the CM criterion for real signals,

$$
J_{\mathrm{CM}}=\frac{1}{4} E\left\{\left(z_{n}^{2}-R_{2}\right)^{2}\right\}
$$

where $z_{n}=\mathbf{f}_{n}^{T} \mathbf{r}_{n}$ is the output of the receiver at time $n$. The dispersion constant $R_{2}$ is equal to unity for binary phase shift keying (BPSK) signals. By minimising $J_{\mathrm{CM}}$ with respect to $\mathbf{f}_{n}^{\mu}$ at a particular step-size $\mu$ and taking the instantaneous gradient, the fixed step-size (fixed- $\mu$ ) CMA receiver weight update equation is given by

The weight update equation of the AS-CMA receiver for real signals is given by [1]

$$
\mathbf{f}_{n+1}=\mathbf{f}_{n}-\mu_{n}\left(z_{n}^{2}-R_{2}\right) z_{n} \mathbf{r}_{n},
$$

where $\mu_{\mathrm{n}}$ is the time-varying step-size. The dispersion constant $R_{2}$ is equal to unity for binary phase shift keying (BPSK) signals. The update of the step-size is given by [1]

$$
\mu_{n+1}=\left[\mu_{n}-\alpha\left(z_{n}^{2}-R_{2}\right) z_{n} Y_{n}^{T} \mathbf{r}_{n}\right]_{\mu_{-}}^{\mu_{+}},
$$

where $[\cdot]_{\mu_{-}}^{\mu_{+}}$denotes truncation to the limits of the range $\left[\mu_{-}, \mu_{+}\right]$ and $\alpha$ denotes the adaptation rate of the step-size $\mu_{n}$ with $\alpha>0$. $Y_{n}$ represents the derivative $\partial \mathbf{f}_{n} /\left.\partial \mu\right|_{\mu=\mu_{n}}$ as defined in [3] and, from (6), the update equation of $Y_{n}$ is given by

$$
Y_{n+1}=\left[\mathbf{I}-\mu_{n}\left(3 z_{n}^{2}-R_{2}\right) \mathbf{r}_{n} \mathbf{r}_{n}^{T}\right] Y_{n}-\left(z_{n}^{2}-R_{2}\right) z_{n} \mathbf{r}_{n},
$$

where I denotes the identity matrix with size $L_{f} \times L_{f}$. Equations (6), (7) and (8) constitute the adaptive step-size CMA algorithm for real signals [1]. It is straightforward to extend these to the complex case, but we retain the real version for consistency with $[4,3]$. For the fixed- $\mu$ system, i.e., $\mu_{n} \triangleq \mu$, we define $Y_{n}^{\mu}=\partial \mathrm{f}_{n}^{\mu} / \partial \mu$ and its update rule is given by

$Y_{n+1}^{\mu}=\left[\mathbf{I}-\mu\left(3\left(z_{n}^{\mu}\right)^{2}-R_{2}\right) \mathbf{r}_{n} \mathbf{r}_{n}^{T}\right] Y_{n}^{\mu}-\left(\left(z_{n}^{\mu}\right)^{2}-R_{2}\right) z_{n} \mathbf{r}_{n}$,

where $z_{n}^{\mu}=\left(\mathbf{f}_{n}^{\mu}\right)^{T} \mathbf{r}_{n}$

\section{INTERPRETATION OF $Y_{N}^{\mu}$ AND $Y_{N}$ OF AS-CMA}

The behaviour of the AS-CMA algorithm is controlled by the random vector parameter $Y_{n}$ defined as the derivative of the estimates of the tap-weight vector with respect to $\mu_{n}$. To study the behaviour of the AS-CMA algorithm, it is essential to understand the meaning of $Y_{n}$. In the algorithm development, although we do not assume that the actual system is stationary, we employ the minimisation of the expectation of a stationary error with respect to $\mu_{n}$.
Thus, we study $Y_{n}$ via $Y_{n}^{\mu}$ which is introduced as a stationary sequence at $\mu_{n} \triangleq \mu$ for the fixed- $\mu$ process. Following [5], a finite difference approximation is used to study the meaning of $Y_{n}^{\mu}$.

Due to the multimodality of the CM cost function, the local minima can be associated with a variety of mean square-error (MSE) values. However, the analysis of [6] has shown a close relationship between the "good" local minima and minimum meansquare error (MMSE) solutions. These local minima are associated with low MSE and are considered as global minima. For a meaningful interpretation of $Y_{n}^{\mu}$, we consider the behaviour of $Y_{n}^{\mu}$ only in the neighbourhood of local minima associated with low MSE.

Following the analysis of [5], $Y_{n}^{\mu}$ is interpreted by considering its derivative representation. For $\Delta>0, \mu+\Delta \leq \mu_{+}$, define $\delta \mathbf{f}_{n}^{\mu, \Delta}=\frac{\mathbf{f}_{a}^{\mu+\Delta}-\mathbf{f}_{n}^{\mu}}{\Delta}$. Therefore,

$$
\begin{aligned}
\delta \mathbf{f}_{n+1}^{\mu, \Delta}= & \frac{1}{\Delta}\left[\mathbf{f}_{n}^{\mu+\Delta}-(\mu+\Delta) \phi\left(z_{n}^{\mu+\Delta}\right) \mathbf{r}_{n}\right. \\
& \left.-\left\{\mathbf{f}_{n}^{\mu}-\mu \phi\left(z_{n}^{\mu}\right) \mathbf{r}_{n}\right\}\right] \\
= & \delta \mathbf{f}_{n}^{\mu, \Delta}-\mu \frac{\left[\phi\left(z_{n}^{\mu+\Delta}\right)-\phi\left(z_{n}^{\mu}\right)\right]}{\Delta} \mathbf{r}_{n}-\phi\left(z_{n}^{\mu+\Delta}\right) \mathbf{r}_{n}
\end{aligned}
$$

where $\phi\left(z_{n}\right)=\left(z_{n}^{2}-R_{2}\right) z_{n}$ is the prediction error function of the CMA algorithm and $z_{n}^{\mu+\Delta}=\left(\mathbf{f}_{n}^{\mu+\Delta}\right)^{T} \mathbf{r}_{n}$. We consider the behaviour of the AS-CMA algorithm within the neighbourhood of an equilibrium point $\tilde{\mathbf{f}}$ in the absence of noise. By using the firstorder Taylor expansion at $z_{n}=\tilde{z}_{n}$ where $\tilde{z}_{n}=\tilde{\mathbf{f}}^{T} \mathbf{r}_{n}$, we arrive at

$$
\begin{aligned}
\phi\left(z_{n}^{\mu+\Delta}\right) & =\phi\left(\tilde{z}_{n}\right)+\phi^{\prime}\left(\bar{z}_{n}\right) \mathbf{r}_{n}^{T}\left(\mathbf{f}_{n}^{\mu+\Delta}-\tilde{\mathbf{f}}\right), \\
\phi\left(z_{n}^{\mu}\right) & =\phi\left(\bar{z}_{n}\right)+\phi^{\prime}\left(\bar{z}_{n}\right) \mathbf{r}_{n}^{T}\left(\mathbf{f}_{n}^{\mu}-\tilde{\mathbf{f}}\right),
\end{aligned}
$$

where $\phi^{\prime}\left(\bar{z}_{n}\right)$ represents the derivative of $\phi\left(\tilde{z}_{n}\right)$ with respect to $\bar{z}_{n}$. Substituting (11) and (12) in (10), we arrive at

$$
\delta \mathbf{f}_{n+1}^{\mu, \Delta}=\delta \mathbf{f}_{n}^{\mu, \Delta}-\mu \phi^{\prime}\left(\tilde{z}_{n}\right) \mathbf{r}_{n} \mathbf{r}_{n}^{T} \delta \mathbf{f}_{n}^{\mu, \Delta}-\phi\left(z_{n}^{\mu+\Delta}\right) \mathbf{r}_{n} .
$$

At $z_{n}=\tilde{z}_{n}$, (9) becomes

$$
Y_{n+1}^{\mu}=Y_{n}^{\mu}-\mu \phi^{\prime}\left(\bar{z}_{n}\right) \mathbf{r}_{n} \mathbf{r}_{n}^{T} Y_{n}^{\mu}-\phi\left(z_{n}^{\mu}\right) \mathbf{r}_{n}
$$

We define $\mathcal{D}_{n}^{\mu, \Delta}=\delta \mathrm{f}_{n}^{\mu, \Delta}-Y_{n}^{\mu}$, therefore, at $z_{n}=\tilde{z}_{n}$,

$$
\begin{gathered}
\mathcal{D}_{n+1}^{\mu, \Delta}=\delta \mathbf{f}_{n}^{\mu, \Delta}-Y_{n}^{\mu}-\mu \phi^{\prime}\left(z_{n}^{\mu}\right) \mathbf{r}_{n} \mathbf{r}_{n}^{T}\left(\delta \mathbf{f}^{\mu, \Delta}-Y_{n}^{\mu}\right) \\
-\phi\left(z_{n}^{\mu+\Delta}\right) \mathbf{r}_{n}+\phi\left(z_{n}^{\mu}\right) \mathbf{r}_{n} \\
=\mathcal{D}_{n}^{\mu, \Delta}-\mu \phi^{\prime}\left(\bar{z}_{n}\right) \mathbf{r}_{n} \mathbf{r}_{n}^{T} \mathcal{D}_{n}^{\mu, \Delta} \\
-\phi^{\prime}\left(\tilde{z}_{n}\right) \mathbf{r}_{n} \mathbf{r}_{n}^{T}\left(\mathbf{f}_{n}^{\mu+\Delta}-\mathbf{f}_{n}^{\mu}\right) \\
=\mathcal{D}_{n}^{\mu, \Delta}-\mu \phi^{\prime}\left(\tilde{z}_{n}\right) \mathbf{r}_{n} \mathbf{r}_{n}^{T} \mathcal{D}_{n}^{\mu, \Delta} \\
-\Delta \phi^{\prime}\left(\tilde{z}_{n}\right) \mathbf{r}_{n} \mathbf{r}_{n}^{T} \delta \mathbf{f}_{n}^{\mu, \Delta},
\end{gathered}
$$

which is in a similar form to $D_{n+1}^{\mu, \Delta}$ shown in Section III of [5]. Similar to the moment bounds of $Y_{n}$ and $Y_{n}^{\mu}$ discussed in Appendix $\mathrm{A}$ of [1], the moment bound for $\delta \mathrm{f}_{n}^{\mu, \Delta}$ is given by $\limsup _{n} E\left\{\left(\delta \mathrm{f}_{n}^{\mu, \Delta}\right)^{2}\right\} \leq O\left(1 / \mu^{2}\right)$. Therefore, as in [5]

$$
\lim \sup _{n} E\left\{\left(\mathcal{D}_{n}^{\mu, \Delta}\right)^{2}\right\}=O(\Delta / \mu) O\left(1 / \mu^{2}\right),
$$

which implies that for the stationary system and small enough $\mu$,

$$
\lim _{\Delta \rightarrow 0} E\left\{\left(Y_{n}^{\mu}-\frac{\mathbf{f}_{n}^{\mu+\Delta}-\boldsymbol{f}_{n}^{\mu}}{\Delta}\right)^{2}\right\}=0 .
$$

Therefore, for the stationary process and in the neighbourhood of the low MSE local minima, $Y_{n}^{\mu}$ retains the interpretation as a derivative in the mean square sense. 


\subsection{Subspace Interpretation of $Y_{n}$ and $Y_{n}^{\mu}$}

We can study the convergence behaviours of $\mathbf{f}_{n}$ in the signal subspace via the weight error vector $\epsilon_{n}=\mathbf{f}_{n}-\tilde{\mathbf{f}}$. It is shown in [2] that mean convergence behaviour of the CMA receiver for DSCDMA near the global minimum of the $\mathrm{CM}$ cost function satisfies

$$
E\left\{\epsilon_{n+1}\right\}=\left(\mathbf{I}-\mu_{n} \sigma_{b}^{2} \mathbf{G} \overline{\mathbf{B}} \mathbf{G}^{T}\right) E\left\{\epsilon_{n}\right\},
$$

where $\sigma_{b}^{2}=E\left\{b_{1}^{2}[n]\right\}$ and $\tilde{\mathbf{B}} \triangleq \frac{1}{\sigma_{b}^{2}} E\left\{\mathbf{b}[n] \phi^{\prime}\left(\bar{z}_{n}\right) \mathbf{b}^{T}[n]\right\}$.

In a similar fashion of studying $Y_{n}$ via $Y_{n}^{\mu}$, we assume the stationary process and study $\epsilon_{n}$ at $\mu_{n} \triangleq \mu$. Defining $\epsilon_{n}^{\mu}=\mathrm{f}_{n}^{\mu}-\overline{\mathrm{f}}$ and $\epsilon_{n}^{\mu+\Delta}=\mathbf{f}_{n}^{\mu+\Delta}-\tilde{\mathbf{f}},(17)$ can be written as

$$
\lim _{\Delta \rightarrow 0} E\left\{\left(Y_{n}^{\mu}-\frac{\epsilon_{n}^{\mu+\Delta}-\epsilon_{n}^{\mu}}{\Delta}\right)^{2}\right\}=0 .
$$

Therefore, $Y_{n}^{\mu}$ also retains the interpretation as a derivative of tap weight error vector in the mean square sense.

By using the eigendecomposition of the Hessian matrix $\mathcal{H} \triangleq$ $\mathbf{G} \bar{B}^{T}{ }^{T}$, we arrive at

$$
\mathcal{H}=\tilde{\mathbf{U}} \tilde{\mathbf{\Lambda}} \tilde{\mathbf{U}}^{T}
$$

where $\tilde{\mathbf{U}}$ is a unitary matrix of normalised eigenvectors and $\tilde{\boldsymbol{\Lambda}}$ is the diagonal matrix of corresponding eigenvalues. Substituting the Hessian matrix $\mathcal{H}$ defined by (20) in (18) and rearranging, we arrive at

$$
E\left\{\epsilon_{n}\right\}=\overline{\mathbf{U}}\left(\mathbf{I}-\mu \sigma_{b}^{2} \tilde{\mathbf{\Lambda}}\right)^{n} \tilde{\mathbf{U}}^{T} \epsilon_{0},
$$

where the expectation operator on $\epsilon_{0}$ is removed since $\epsilon_{0}$ is not considered a random vector. At $\mu_{n}=\mu$ and $\mu+\Delta$.

$$
\begin{aligned}
E\left\{\boldsymbol{\epsilon}_{n}^{\mu}\right\} & =\tilde{\mathbf{U}}\left(\mathbf{I}-\mu \sigma_{b}^{2} \tilde{\mathbf{\Lambda}}\right)^{n} \tilde{\mathbf{U}}^{T} \epsilon_{0}, \\
E\left\{\epsilon_{n}^{\mu+\Delta}\right\} & =\tilde{\mathbf{U}}\left(\mathbf{I}-(\mu+\Delta) \sigma_{b}^{2} \tilde{\mathbf{\Lambda}}\right)^{n} \tilde{\mathbf{U}}^{T} \epsilon_{0},
\end{aligned}
$$

where $\epsilon_{0}^{\mu+\Delta}=\epsilon_{0}^{\mu}=\epsilon_{0}$. The signal subspace eigenvectors form the column of matrix $\overline{\mathbf{U}}_{s}$ and the noise subspace ones form the matrix $\tilde{\mathbf{U}}_{n}$, i.e., $\tilde{\mathbf{U}}=\left[\tilde{\mathbf{U}}_{s} \tilde{\mathbf{U}}_{n}\right]$. It is shown that the MMSE solution $\mathrm{f}_{\mathrm{MMSE}}$ lies in the signal subspace [7]. For the binary input, it is shown in [8] that $\overline{\mathbf{f}}$ coincides with $\mathbf{f}_{\mathrm{MMSE}}$ implying that $\tilde{\mathbf{U}}_{n}^{T} \overline{\mathbf{f}}=$ 0 . Therefore, if $\mathrm{f}_{0}$ also lies in the signal subspace, $\overline{\mathrm{U}}_{n}^{T} \epsilon_{0}=\mathbf{0}$. Hence, from (22) and (23), we arrive respectively at

$$
\begin{aligned}
E\left\{\epsilon_{n}^{\mu}\right\} & =\tilde{\mathbf{U}}_{s}\left(\mathbf{I}-\mu \sigma_{b}^{2}\left(\tilde{\boldsymbol{\Lambda}}_{s}-\sigma_{w}^{2} \mathbf{I}\right)\right)^{n} \tilde{\mathbf{U}}_{s}^{T} \epsilon_{0} \\
E\left\{\boldsymbol{\epsilon}_{n}^{\mu+\Delta}\right\} & =\tilde{\mathbf{U}}_{s}\left(\mathbf{I}-(\mu+\Delta) \sigma_{b}^{2}\left(\tilde{\boldsymbol{\Lambda}}_{s}-\sigma_{w}^{2} \mathbf{I}\right)\right)^{n} \tilde{\mathbf{U}}_{s}^{T} \boldsymbol{\epsilon}_{0}
\end{aligned}
$$

where $\tilde{\boldsymbol{\Lambda}}_{s}$ denotes the diagonal matrix of nonzero eigenvalues of $\mathcal{H}$.

Setting $\mathrm{f}_{0}^{\mu}$ in the signal subspace implies faster convergence speed for both the least-mean-square (LMS) algorithm [9] and CMA [2]. From (19), (24) and (25), it is shown that $Y_{n}^{\mu}$ can be bounded within the signal subspace provided that the initialisation $\mathrm{f}_{\sigma}^{\mu}$ is in the signal subspace. This bounding of $Y_{n}^{\mu}$ implies that the convergence speed and time constant of the algorithm can be assessed by considering only the eigenvalues associated with the eigenvectors spanning the signal subspace $[2,9]$.

\section{NUMERICAL RESULTS}

We considered a synchronous DS-CDMA system with spreading gain $31(N=31)$. Without loss of generality, the first user was the desired user with unity power. The background noise was zero mean $A W G N$ with $S N R=20 \mathrm{~dB}$ referenced to the desired user. We considered the performance of adaptive step-size receivers in a dynamic environment where different user groups switched between three user groups according to a Markov chain. The dynamic environment was set similarly to that of [3] with minor modification. The power of the first user was set such that its SNR was 20 $\mathrm{dB}$ and the multiple-access interference (MAI) user groups were designated as follows. User group 1 consisted of $17 \mathrm{MAI}$ users transmitting at $20 \mathrm{~dB}$ and two MAI users transmitting at $30 \mathrm{~dB}$. User group 2 consisted of 26 MAI users transmitting at $20 \mathrm{~dB}$. Finally, user group 3 consisted of eight MAI users transmitting at 20 $\mathrm{dB}$ and one MAI user transmitting at $30 \mathrm{~dB}$. The MAI structure was constructed according to a Markov chain $x[n]$ with transition probability matrix

$$
\mathbf{P}=\left[\begin{array}{ccc}
0.998 & 0.01 & 0.01 \\
0.01 & 0.998 & 0.01 \\
0.01 & 0.01 & 0.998
\end{array}\right]
$$

and state space $\left\{(1,0,0)^{T},(0,1,0)^{T},(0,0,1)^{T}\right\}$. The Markov chain $x[n]$ designates the MAI user group which affects the desired user detection at the $n$th symbol. The switching between the three MAI user groups occurred at every 500 symbols. Five-ray multipath fading channels were used for both the desired user and all the MAI users for all user groups. The last four rays were uniformly distributed in delay over $\left[0,10 T_{c}\right)$, where $T_{c}$ is the chip interval, with standard deviation 0.3 . Averaged SINR was used as a performance measure and all plots were averaged over 50 MonteCarlo runs.

We compare the SINR performances of the AS-CMA receiver, the AS-MOE receiver [3] and the AS-SA receiver [5]. All the receiver lengths were chosen to be twice that of the spreading gain. For all AS algorithms, the adaptation rates $\alpha$ were $10^{-7}$ and the upper and lower step-size limits $\mu_{+}, \mu_{-}$were set respectively at $10^{-2}$ and 0 . The averaged SINR plots of all algorithms are compared in Fig. 1. Notice that while both the AS-SA and AS-CMA receivers have an ability to track variations of multipath channels, the AS-MOE receiver fails to operate due to mismatch. The SINR plot of AS-CMA almost coincides with that of AS-SA indicating the comparable performance of the two algorithms.

In Fig. 2, the steady-state SINR of the AS-CMA receiver for different initial settings of step-sizes $\mu_{0} \in\left[10^{-8}, 10^{-3}\right]$ are compared with that of the AS-SA receiver. Despite a 100000 -fold variation in $\mu_{0}$, it is shown that the SINR performances of both receivers are maintained at a constant level. The AS-CMA is also shown to achieve a comparable SINR performance as the AS-SA. This result is attributed to the excellent performance of the CM criterion which has been shown to be near the Wiener receiver $[10,6]$. Since the SINR results of AS-MOE are approximately at $-12 \mathrm{~dB}$ throughout the setting range of $\mu_{0}$, AS-MOE is therefore discarded in the plot.

Fig. 3 shows the trajectories of $\mu_{n}$ of the AS-CMA receiver and the AS-SA at three different settings of the initial step-size $\mu_{0}=\left\{10^{-8}, 10^{-5}, 10^{-3}\right\}$. The trajectories were plotted for 80000 symbols. Insensitivity to the settings of the initial step-size for each algorithm can be observed. The adaptation behaviour of $\mu_{n}$ 


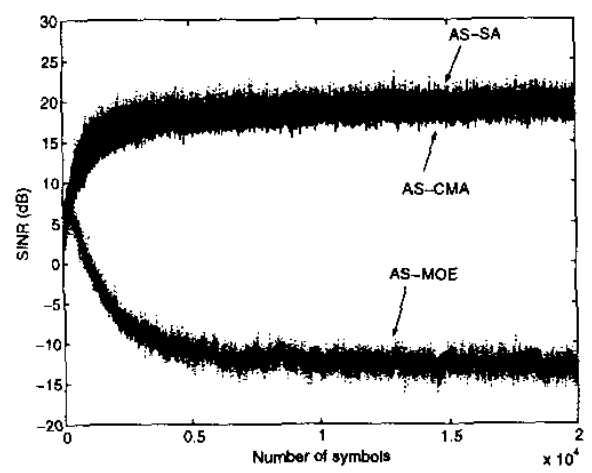

Figure 1: The SINR performance of AS-SA [5], AS-MOE [3] and AS-CMA receivers in a Markovian dynamic environment. The AS-MOE receiver fails to operate due to mismatch. AS-SA and AS-CMA are comparable in terms of convergence speed and steady-state performance.

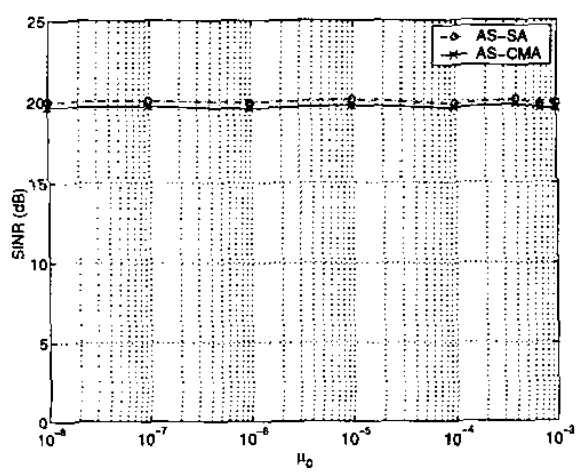

Figure 2: SINR comparison of AS-SA and AS-CMA at different settings of initial stepsizes $\mu_{0}$. It is shown that both AS-SA and AS-CMA are not affected by various initial settings despite 100000 -fold of variation of $\mu_{0}$. Notice an almost identical performance of both algorithms.

for AS-CMA is similar to that of AS-SA. The convergence speed of both algorithms are approximately the same.

\section{CONCLUSION}

Convergence behaviours of the adaptive step-size CMA algorithm for a DS-CDMA receiver are studied. Analysis reveals similarity in convergence behaviours of the $Y_{n}$ process of AS-CMA and ASSA. Simulation shows the effectiveness of the AS-CMA algorithm in both interference cancellation and channel equalisation for a nonstationary multipath fading channel. A close relation in the step-size trajectories between the blind AS-CMA algorithm and the non-blind AS-SA algorithm is demonstrated. Robustness to variation of the initial settings of the step-size of AS-CMA is also shown.

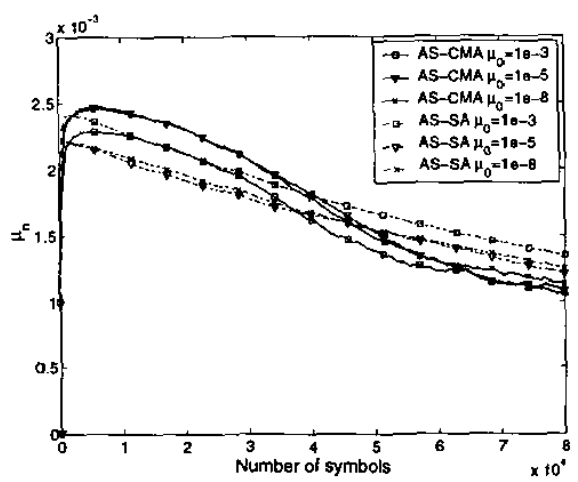

Figure 3: Trajectories of $\mu_{n}$ for AS-SA and AS-CMA at different settings $\mu_{0}=\left\{10^{-8}, 10^{-5}, 10^{-3}\right\}$. Insensitivity to variation in the initial step-size settings for each algorithm can be observed. Similarity in adaptation behaviours of $\mu_{n}$ of both algorithms is shown.

\section{REFERENCES}

[1] P. Yuvapoositanon and J. A. Chambers, "Adaptive step-size constant modulus algorithm for DS-CDMA receivers in nonstationary environments," Signal Processing, vol. 82, pp. 311-315, 2002.

[2] P. Yuvapoositanon, Blind Adaptive Techniques for DirectSequence Code Division Multiple Access Receivers, Ph.D. thesis, Imperial College, University of London, England, 2002.

[3] V. Krishnamurthy, G. Yin, and S. Singh, "Adaptive step-size algorithms for blind interference suppression in DS/CDMA systems," IEEE Trans. Signal Processing, vol. 49, no. 1, pp. 190-201, 2001.

[4] M. L. Honig, U. Madhow, and S. Verdú, "Blind adaptive multiuser detection," IEEE Trans. Inform. Theory, vol. 41, no. 4, pp. 944-966, 1995.

[5] H. J. Kushner and J. Yang, "Analysis of adaptive step-size SA algorithms for parameter tracking," IEEE Trans. Automat. Contr, vol. 40, no. 8, pp. 1403-1410, 1995.

[6] H. H. Zeng, L. Tong, and C. R. Johnson Jr., "Relationships between the constant modulus and Wiener receivers," IEEE Trans. Inform. Theory, vol. 44, no. 4, pp. 1523-1538, 1998.

[7] X. Wang and H. V. Poor, "Blind multiuser detection: A subspace approach," IEEE Trans. Inform. Theory, vol. 44, no. 2, pp. 677-690, 1998.

[8] Y. Li and K. J. R. Liu, "Static and dynamic convergence behavior of adaptive blind equalizers," IEEE Trans. Signal Processing, vol. 44, no. 11, pp. 2736-2745, 1996.

[9] T. J. Lim, Y. Gong, and B. Farhang-Boroujeny, "Convergence analysis of chip- and fractionally spaced LMS adaptive multiuser CDMA detectors," IEEE Trans. Signal Processing, vol. 48, no. 8, pp. 2219-2228, 2000.

[10] C. R. Johnson Jr., P. Schniter, T. J. Endres, J. D. Behm, D. R. Brown, and R. A. Casas, "Blind equalization using the constant modulus criterion; A review," Proc. IEEE, vol. 86, no. 10, pp. 1927-1950, 1998. 